\title{
Doppler Ultrasound Evaluation of the Efficacy of High Intensity Focused Ultrasound in Treatment of Cesarean Section Scar Pregnancy
}

\author{
Xiaokai Huo ${ }^{1}$, Yaozeng Xie ${ }^{2}$, Lei Yang², Kun Deng² and Yingying Liu² \\ ${ }^{1}$ Department of Ultrasonography, The Second people's Hospital of Liaocheng City Affiliated to Shandong First Medical University, \\ Shandong Province, China \\ ${ }^{2}$ Department of imaging, The Second people's Hospital of Liaocheng City Affiliated to Shandong First Medical University, \\ Shandong Province, China
}

\begin{abstract}
Objective: To investigate the value of Doppler ultrasound in evaluating the efficacy of high intensity focused ultrasound (HIFU) in treatment of cesarean section scar pregnancy.

Study Design: Observational study.

Place and Duration of Study: Department of Ultrasonography, The Second people's Hospital of Liaocheng City Affiliated to Shandong First Medical University, China, from January 2015 to December 2017.

Methodology: Forty-six patients with cesarean section scar pregnancy treated with HIFU were enrolled. Doppler ultrasound images, blood flow resistance index (RI) and $\beta$-human chorionic gonadotropin ( $\beta$-hCG) levels were analyzed. Results: Among 46 patients, 31 cases (68.89\%) were embryo sac type while 15 cases (32.61\%) were mass-type. After 4 weeks of HIFU treatment, among 31 cases of embryo sac type of cesarean scar pregnancy, slightly increased echogenicity and thickened walls of yolk sac were observed in 15 cases (48.39\%), heart beat disappeared in 12 cases $(38.71 \%)$ and fetal heart rate as still regular in four cases $(12.90 \%)$. Among 15 cases of mass-type treated with 4 weeks of HIFU, echogenicity was slightly elevated at first, then echogenicity of center zone decreased, resulting in mixed echoes, finally the volumes of mass decreased to varying degrees and blood flow reduced significantly, leading to slight strong mull echoes. Compared with before treatment of HIFU, RI levels were higher after 4 weeks treatment of HIFU $(p<0.001)$, while the blood $\beta$-hCG levels were lower $(p<0.001)$.

Conclusion: Doppler ultrasound can provide accurate qualitative diagnosis of cesarean section scar pregnancy, and possess higher clinical value in evaluating the treatment efficacy of cesarean section scar pregnancy with HIFU.
\end{abstract}

Key Words: Doppler ultrasound, Cesarean section, Pregnancy, Ectopia, High intensity focused ultrasound, Efficacy.

How to cite this article: Huo X, Xie Y, Yang L, Deng K, Liu Y. Doppler ultrasound evaluation of the efficacy of high intensity focused ultrasound in treatment of cesarean section scar pregnancy. J Coll Physicians Surg Pak 2020; 30(3):240-244.

\section{INTRODUCTION}

Cesarean section scar pregnancy, a special type of ectopic pregnancy, refers that most or all of fertilized eggs are implanted at the incision scar (lower segments of uterine anterior wall) after cesarean section..$^{1,2}$ Cesarean section scar pregnancy usually manifests as irregular vaginal bleeding, but uterine rupture may occur as it worsens, leading to massive bleeding and a lifethreatening condition. ${ }^{3}$ At present, there are many diagnostic ways of cesarean section scar pregnancy, including hysteroscope, ultrasound and MRI. However, hysteroscope is not suitable for patients with vaginal

Correspondence to: Dr. Xiaokai Huo, Department of

Ultrasonography, The Second people's Hospital of Liaocheng

City Affiliated to Shandong First Medical University, Shandong

Province, 252600, China

E-mail: eakec4@163.com

Received: May 22, 2018; Revised: October 24, 2019,

Accepted: February 19, 2020 bleeding as it may even cause increased bleeding, and $\mathrm{MRI}$ is rather expensive. ${ }^{4}$ Ultrasonography has received increasing attention in recent years. A study found ultrasonography was feasible in the diagnosis of ectopic pregnancy. ${ }^{5}$

The treatment of cesarean section scar pregnancy is diverse, but no unified standard was developed. ${ }^{6}$ With the gradual promotion of non-invasive techniques, high intensity focused ultrasound (HIFU), a new treatment method, has been applied to the clinical treatment of scar pregnancy. ${ }^{7}$ However, there is no clear and unified criterion for evaluating the short-term efficacy of HIFU in the treatment of scar pregnancy. Sterzik et al. demonstrated the high predictive value of serum $\beta$-human chorionic gonadotropin ( $\beta-\mathrm{hCG}$ ) determinations in diagnosis of early ectopic pregnancies. ${ }^{8}$ Baradwan et al. revealed it was a successful response to treatment of viable cesarean scar twin pregnancy was monitored by $\beta$-hCG and ultrasound. ${ }^{9}$ 
The aim of this study was to compare Doppler ultrasound and characteristics of ultrasound and $\mathrm{RI}$ levels before and after treatment and $\beta$-hCG levels on patients with cesarean section scar pregnancy, for evaluation of treatment of cesarean section scar pregnancy with HIFU.

\section{METHODOLOGY}

This study was conducted at the Department of Ultrasonography, The Second people's Hospital of Liaocheng City Affiliated to Shandong First Medical University, China, from January 2015 to December 2017, after approval by the Institutional Ethics Committee of Hospital. A total of 46 patients with cesarean section scar pregnancy were enrolled. Inclusion criteria were patients with more than 2 years had passed since the patient last had cesarean section, where cesarean section scar pregnancy was identified by ultrasound results, and serum $\beta$-hCG. Exclusion criteria were patients with malignant tumor or blood system disease, and patients with cardiac, cerebral, hepatic or renal dysfunction.

All patients were treated with HIFU. JC 200 Focused ultrasound instrument for tumor therapy from Chongqing Medical Technology Corporation Limited were purchased. Before treatment, all patients were under diet control and prepared for clyster. During the treatment, patients were placed in prone position, with proper filling of bladder. Intestinal was pressed with proper instruments. Sedation and analgesia were performed after safe access was built. First identify the range and level of treatment, with frequency set at $0.85 \mathrm{~Hz}$, fixation time at 1-2 second, break at 2-6 second and power at 300-400 W. At the beginning and ending of treatment, sulphur hexafluoride microbubbles imaging was performed immediately. During the whole process, the radiation area, power, radiation time and interval time were adjusted according to patients' reaction. The entire treatment process is monitored by ultrasound in real time.

Before and after 4-week of treatment of HIFU, examination on patients were performed by Philips IU22 Ultrasound Instrument, with the frequency of convex array probe set at 3.5 - 5.5 MHz, and C5-1 virginal probe at $7-13 \mathrm{MHz}$. Size of gestational sac or mass, size of embryo and yolk sac, original heart beats, morphology, edge and internal echoes of mass, myometrial thickness at the site of scar and blood flow in the muscle layer of peri - gestational sac or lesion were observable via combination of transvaginal ultrasound and transabdominal ultrasound with patients at supine position and lithotomy position. Both left and right side of uterine artery trunk were monitored from the distal branch of internal iliac artery. Three consecutive stable Doppler spectrum were obtained and $\mathrm{RI}$ was recorded.
Examinations on all patients were performed by one sophisticated physicians. The blood $\beta$-hCG levels were detected by enzyme-linked immunosorbent assay (ELISA).

Classification standards of cesarean section scar pregnancy included embryo sac type and mass-type. Embryo sac type was defined by typical double ring sign was observed in the site of scar while yolk sac, embryo, or fetal heart beats were visible in some cases. Masstype was defined by mass-type, patchy mixed echoes were visible in the site of scar, where normal myometrium was thin or disappeared, with a blur boundary between incision and muscular layer.

Grading standards of blood flow included rich blood flow, slightly rich blood flow, low blood flow, and no blood flow. Rich blood flow was observed in more than two vessels, with more than 4 dotted blood flow signals. Slightly rich blood flow was observed in one or two vessels, with 3-4 dotted blood flow signals. Low blood flow was observed in 1-2 dotted blood flow signals. No blood flow was observed in zero blood flow signals.

Data were analysed using spss version 25 . Mean value $\pm S D$ was calculated for numerical variables like $\mathrm{RI}$ and $\beta$-hCG levels, etc. and compared by paired t-test. Frequencies and percentages were calculated for categorical variables. The $p$-values less than 0.05 were regarded as significant.

\section{RESULTS}

All patients were female, the average age was 30.61 \pm 2.54 years, ranging from 28 to 37 years. The average pregnant frequency was $3.7 \pm 0.5$ times, ranging from 2 to 7 times. The average time since the patient last had cesarean section was $4.67 \pm 1.38$ years, ranging from 1 to 10 years.

Results from Doppler ultrasound showed that of 46 patients, the 31 of cases $(68.89 \%)$ of them were embryo sac type of cesarean scar pregnancy while 15 cases $(32.61 \%)$ were mass-type. After 4 weeks of HIFU treatment, among the 31 of cases embryo sac type of cesarean scar pregnancy, slightly increased echogenicity and thickened walls of yolk sac were observed in 15 cases $(48.39 \%)$, original heart beats disappeared in 12 cases $(38.71 \%)$ and fetal heart rates were still regular in 4 cases $(12.90 \%)$. Among the 15 cases masstype of cesarean scar pregnancy treated with 4 weeks of HIFU, echogenicity was slightly elevated at first, then echogenicity of center zone decreased, resulting in mixed echoes, finally the volumes of mass decreased to varying degrees and blood flow reduced significantly, leading to slight strong mull echoes.

Before treatment of HIFU, abnormally rich garland-like blood signals of red and blue colors and arteriovenous fistula-like spectrum were observed in 27 cases 
Table I: Changes of RI, blood $\beta$-hCG levels were measured before and after 4-week treatment of HIFU.

\begin{tabular}{l|c|cc|c}
\hline Time & $\mathrm{n}$ & & $\mathrm{RI}$ & $\mathrm{B}$ \\
\cline { 3 - 5 } & & Mean \pm SD & $\mathrm{p}$-value & $\mathrm{M}$ \\
\hline Before treatment of HIFU & 46 & $0.35 \pm 0.06$ & $<.001$ & $3798.16 \pm 185.74$ \\
After 4-week treatment of HIFU & 46 & $0.63 \pm 0.05$ & & $125.16 \pm 9.75$ \\
\hline
\end{tabular}

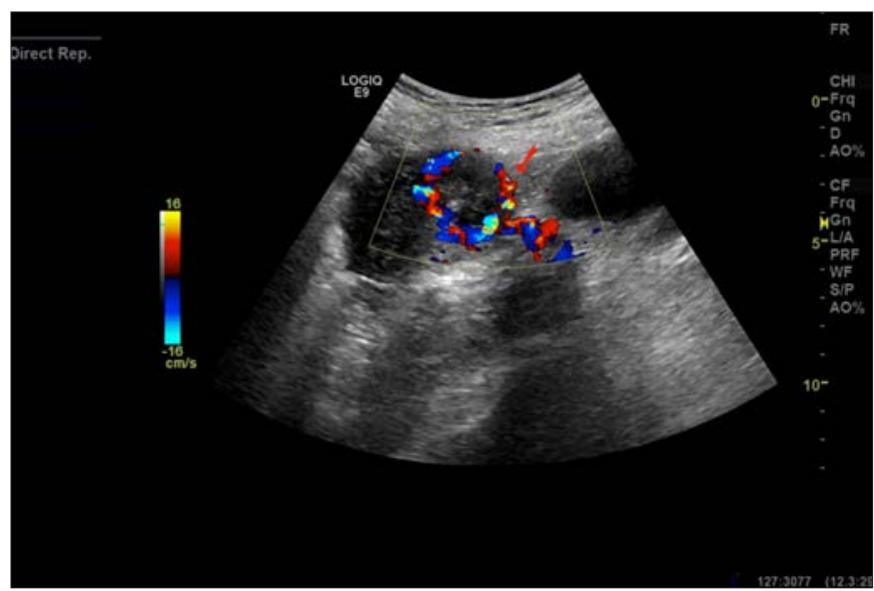

Figure 1: Before the HIFU treatment, rich garland-like blood signals around the gestational sac.

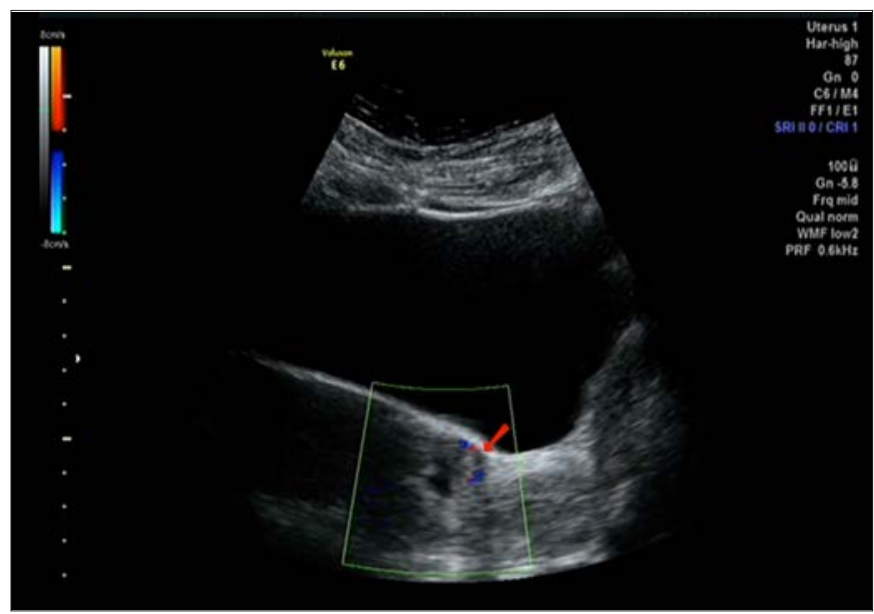

Figure 2: After 4-week treatment of HIFU, only dotted blood flow signals were observed in the site of scar.

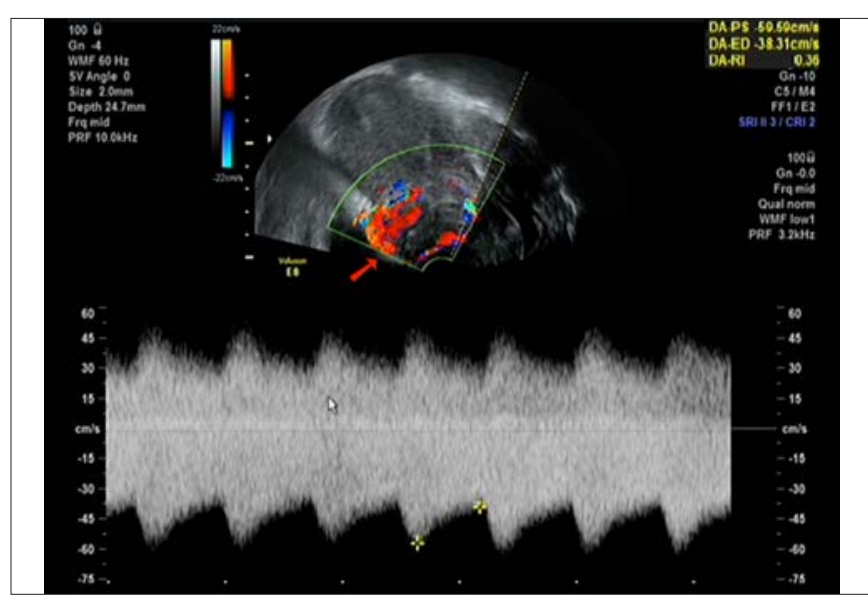

Figure 3: Before the HIFU treatment, blood signals were observed in the mass-type cesarean scar pregnancy, with low RI.

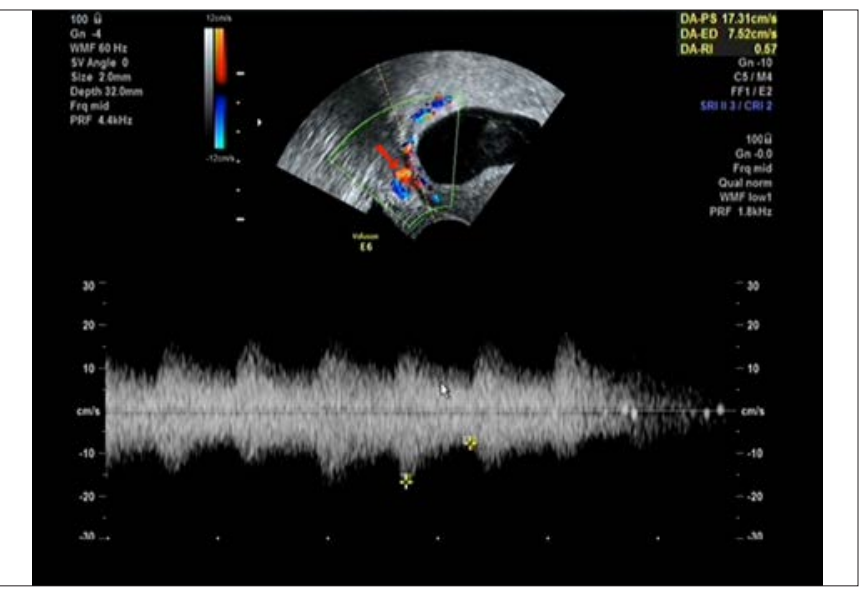

Figure 4: After 4-week treatment of HIFU, deformed yolk sac was observed in cesarean scar pregnancy patients, with poor blood flow but higher RI around the gestational sac.

$(58.70 \%$, Figure 1). And poor or no signal of blood flow was observed in those patients after 4-week treatment of HIFU (Figure 2). Among 46 patients, 14 cases (30.43\%) of them presented with slightly rich blood flow signal before the HIFU treatment. Poor or no signal of blood flow and the RI levels were observed in those patients after 4-week treatment of HIFU (Figures 3 and 4). Among 46 patients, 5 cases $(10.87 \%)$ of them presented with poor blood flow signal before the HIFU treatment. But no significant change was observed after 4-week treatment of HIFU.

Compared with before treatment of HIFU, RI levels were significantly higher after 4-week treatment of HIFU $(p<0.001)$, while the blood $\beta$-hCG levels were significantly lower $(p<0.001$, Table I).

\section{DISCUSSION}

Cesarean section scar pregnancy is a special type of ectopic pregnancy, which could be related to poor incision healing after cesarean section, endometrium injury during surgery, excessive movement of fertilized eggs, infiltration of trophoblast into the scar, etc., leading to uterine rupture or hemorrhagic shock. ${ }^{10,11}$ The main purposes of the treatment of cesarean section scar pregnancy are terminating pregnancy, preventing massive blood loss or uterine rupture during curettage and evacuation of uterus and conserving fertility. ${ }^{12,13}$ Treatment with medication only has longer recovery time and poor efficacy. Traditional direct curettage and evacuation of uterus could result in complications like massive blood loss, uterine rupture, or bladder injury. Interventional treatment of uterine artery could cause 
embolism in bilateral uterine artery, reduce blood supply and provide better chances for curettage and evacuation of uterus. However, it could also cause postembolism syndrome like lower abdominal pain, fever, nausea and vomiting, massive blood loss and enlargement of scar size after second surgery, increasing the risks of next cesarean section scar pregnancy. ${ }^{14,15}$ Hysterectomy does severe damage to the reproductive organs, affecting physical and mental health. Hence a safer and more effective approach is desired. Application of HIFU in the treatment of cesarean section scar pregnancy has considerable prospects. The underlying mechanisms involves application of high-intensity ultrasound energy (focus) on the embryos or masses in the gestational sac and peripheral blood vessels (nourishing blood vessels), locally generating transient high temperature (above $65^{\circ}$ ), and induction of coagulation necrosis via biological effects such as high temperature, cavitation, mechanical action, and biological immunity, achieving therapeutic purposes. ${ }^{16,17}$

With the development of high-resolution ultrasound diagnostic apparatus, the images of the occurrence of ectopic pregnancy in the early uterus are more clear. ${ }^{18}$ The condition of cervical and lower uterine segment, the relative positions between gestational sac and incision are more accurately shown in transvaginal ultrasound. ${ }^{19}$ The relative positions between gestational sac and bladder, and the thickness of uterine serosa are more accurately shown in transabdominal ultrasound. ${ }^{20}$ Combination of transvaginal ultrasound and transabdominal ultrasound help thoroughly understand both partial and whole picture of gestational sac or lesion. And the measurement of gestational sac and RI level and provide accurate data for clinical treatment.

Results from the present study proved that $\beta-h C G$ is an effective indicator of scar pregnancy treatment efficacy, but it could not provide updated reflection of lesion. ${ }^{21}$

Changes in lesion and blood flow can be directly observed by Doppler ultrasound. Single application of either $\beta$-hCG or Doppler ultrasound has limitations. Results from our study showed that RI level was relatively low while $\beta$-hCG was relatively high in patients before HIFU treatment. And RI level significantly increased while $\beta$-hCG significantly decreased in patients after 4-week of HIFU treatment. Another study confirmed that HIFU was able to stop embryonic cardiac activity and leaded to a rapid reduction in serum human chorionic gonadotropin levels. ${ }^{22}$ This study showed that combination of RI measurement with Doppler ultrasound and blood $\beta$-hCG played significant indicator roles in HIFU treatment evaluation. The findings of this study were similar to those reported by Buresch et al. ${ }^{23}$

There were some limitations in this study, such as small sample size and single-observation index. However, this study brings a new perspective for larger sample-sized studies, considering the Doppler ultrasound technique is feasible and applicable.

\section{CONCLUSION}

Doppler ultrasound can provide accurate qualitative diagnosis of cesarean section scar pregnancy, and possess higher clinical value in in evaluating the treatment efficacy of cesarean section scar pregnancy with HIFU.

\section{ETHICAL APPROVAL:}

The study was approved prior to initiation of the research work by the Ethics Committees of The Second people's Hospital of Liaocheng City Affiliated to Shandong First Medical University, China.

\section{PATIENTS' CONSENT:}

Informed consents were obtained from all patients.

\section{CONFLICT OF INTEREST:}

Authors declared no conflict of interest.

\section{AUTHORS' CONTRIBUTION:}

$\mathrm{XH}$ : Responsible for revising it critically for important intellectual content and the final approval of the version to be published.

YX, LY: Responsible for the analysis of data for the work. $\mathrm{KD}, \mathrm{YL}$ : Responsible for the conception and design of the work.

\section{REFERENCES}

1. Timor-Tritsch IE, Monteagudo A. Unforeseen consequences of the increasing rate of cesarean deliveries: Early placenta accreta and cesarean scar pregnancy. A review. Am J Obstet Gynecol 2012; 207:14-29.

2. Chao A, Wang TH, Wang CJ, Lee CL, Chao AS. Hysteroscopic management of cesarean scar pregnancy after unsuccessful methotrexate treatment. J Minim Invasive Gynecol 2005; 12 : 374-6.

3. Sugawara J, Senoo M, Chisaka H, Yaegashi N, Okamura K. Successful conservative treatment of a cesarean scar pregnancy with uterine artery embolization. Tohoku $J$ Exp Med 2005; 206:261-5.

4. Ayala YÃR, Briones LC, Anaya CH, Leroy LL. Diagnosis of molar pregnancy by hysteroscopy. Ginecol Obstet Mex 2012; 80:540-4.

5. Madani Y. The use of ultrasonography in the diagnosis of ectopic pregnancy: A case report and review of the literature. Medscape J Med 2008; 10:35.

6. Tulpin L, Morel O, Malartic C, Barranger E. Conservative management of a cesarean scar ectopic pregnancy: A case report. Cases J 2009; 2:7794.

7. Zou M, Chen L, Wu C, Hu C, Xiong Y. Pregnancy outcomes in patients with uterine fibroids treated with ultrasound-guided high-intensity focused ultrasound. Bjog 2017; 124:30-5.

8. Sterzik K, Rosenbusch B, Benz R. Serum specific protein 1 and beta-human chorionic gonadotropin concentrations in patients with suspected ectopic pregnancies. Int J Gynaecol Obstet 1989; 28:253-6. 
9. Baradwan S, Khan F, Al-Jaroudi D. Successful management of a spontaneous viable monochorionic diamniotic twin pregnancy on cesarean scar with systemic methotrexate: A case report. Medicine (Baltimore) 2018; 97:e12343.

10. Wang DB, Chen YH, Zhang ZF, Chen P, Liu KR, Li Y, et al. Evaluation of the transvaginal resection of low-segment cesarean scar ectopic pregnancies. Fertil Steril 2014; 101: 602-6.

11. Biyagama BR, Fernando A, Silva KC, Kobalakrishnan M, Kobalakrishnan M, Wijenayake UN. Laparoscopic excision of caesarean scar ectopic pregnancy. Ceylon Med J 2015; 60: 111-2.

12. Sun YY, Xi XW, Yan Q, Qiao QQ, Feng YJ, Zhu YP. Management of type II unruptured cesarean scar pregnancy: comparison of gestational mass excision and uterine artery embolization combined with methotrexate. Taiwan J Obstet Gynecol 2015; 54:489-92.

13. Juneja SK, Tandon P, Chopra I. Successful pregnancy after excision of cesarean scar endometriosis with uterovesicocutaneous fistula: A rare case report. Int J Appl Basic Med Res 2016; 6:300-2.

14. Taehwan K, Hoon SJ, Jinoo K, Hyun-Ki Y, Gi-Young K, Dong-II G, et al. Management of bleeding uterine arteriovenous malformation with bilateral uterine artery embolization. Yonsei Med $\mathrm{J}$ 2014; 55:367-73.

15. Bratby MJ, Ramachandran N, Sheppard N, Kyriou J, Munneke GM, Belli AM. Prospective study of elective bilateral versus unilateral femoral arterial puncture for uterine artery embolization. Cardiovasc Intervent Radiol 2007; 30:1139-43.
16. Yu L, Xu L, Xu X. Treatment of cornual pregnancy in a patient with adenomyosis by high-intensity focused ultrasound (HIFU) ablation: A case report. Medicine 2017; 96:e8874.

17. Marinova M, Rauch M, Schild HH, Strunk HM. Novel noninvasive treatment with high-intensity focused ultrasound (HIFU). Ultraschall in Der Medizin 2015; 37:46-55.

18. Mutihir JT, Nyango DD. Massive haemoperitoneum from endometriosis masquerading as ruptured ectopic pregnancy: Case report. Niger J Clin Pract 2010; 13:477-9.

19. Marasinghe JP, Senanayake H, Randeniya C, Seneviratne HR, Arambepola C, Devlieger R. Comparison of transabdominal versus transvaginal ultrasound to measure thickness of the lower uterine segment at term. Int J Gynaecol Obstet 2009; 107:140-2.

20. Lin CY, Shih YT. Transabdominal ultrasound-guided intragestational sac single dose $\mathrm{mtx}$ injection in a tubal pregnancy. Taiwan J Obstet Gynecol 2010; 49:536-8.

21. Muraji M, Mabuchi S, Hisamoto K, Muranishi M, Kanagawa T, Nishio $\mathrm{Y}$, et al. Cesarean scar pregnancies successfully treated with methotrexate. Acta Obstet Gynecol Scand 2009; 88:720-3.

22. Huang L, Du Y, Zhao C. High-intensity focused ultrasound combined with dilatation and curettage for Cesarean scar pregnancy. Ultrasound Obst Gyn 2014; 43:98-101.

23. Buresch AM, Chavez MR, Kinzler W, Vintzileos AM. Use and value of ultrasound in diagnosing cesarean scar pregnancy: A report of three cases. J Reprod Med 2014; 59:517-21. 